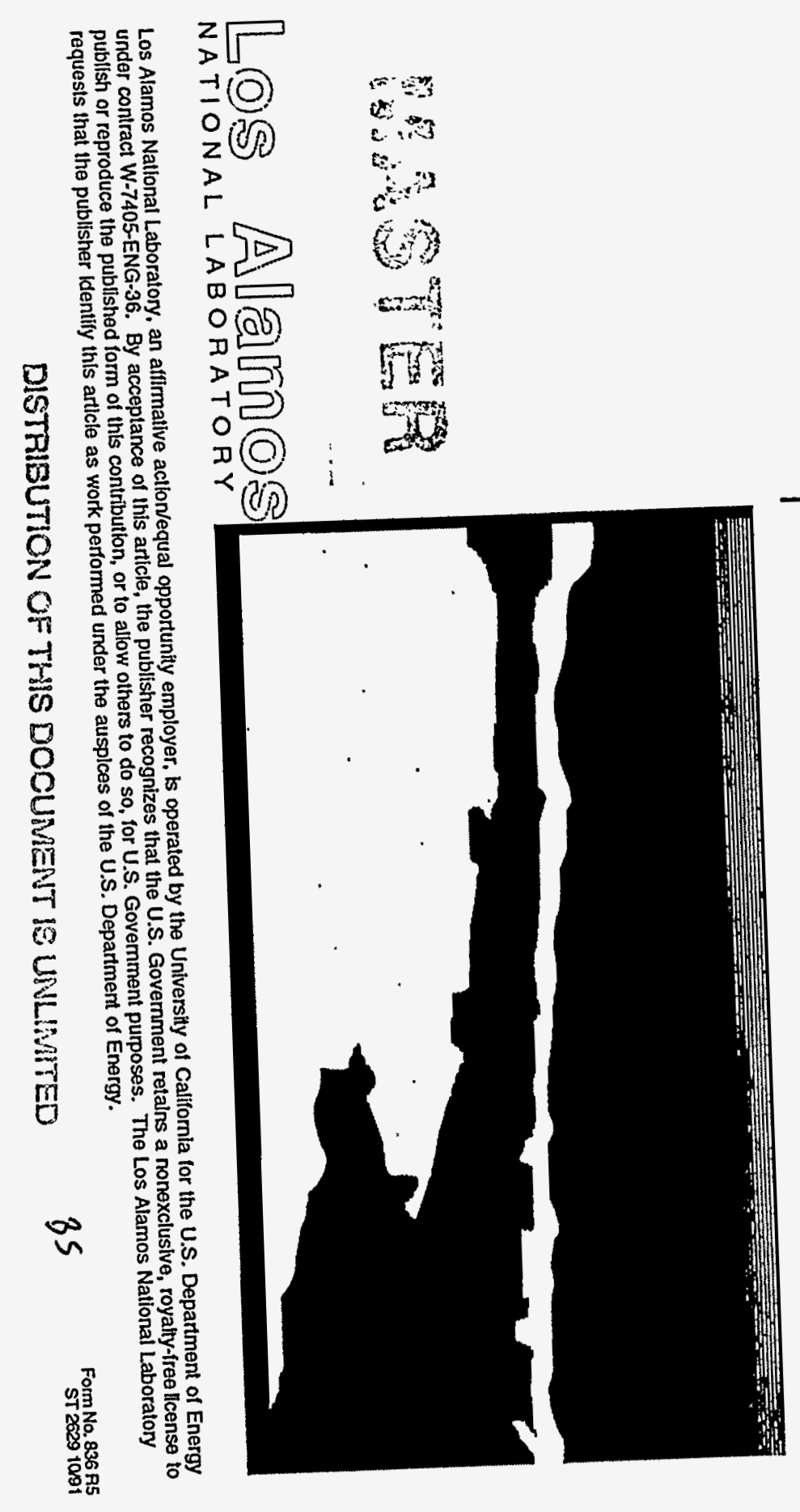

\section{DISCLAIMER}

This report was prepared as an account of work sponsored by an agency of the United States Government. Neither the United States Government nor any agency thereof, nor any of their employees, makes any warranty, express or implied, or assumes any legal liability or responsibility for the accuracy, completeness, or usefulness of any information, apparatus, product, or process disclosed, or represents that its use would not infringe privately owned rights. Reference herein to any specific commercial product, process, or service by trade name, trademark, manufacturer, or otherwise does not necessarily constitute or imply its endorsement, recommendation, or favoring by the United States Government or any agency thereof. The views and opinions of authors expressed herein do not necessarily state or reflect those of the United States Government or any agency thereof. 


\title{
Flare Star Observations with a Single-Photon Counting Imaging Detector
}

\author{
Donald E. Casperson, William C. Priedhorsky, Miles H. Baron, and Cheng Ho
}

\author{
Astrophysics and Radiation Measurements Group NIS-2 \\ Los Alamos National Laboratory \\ Los Alamos, New Mexico, 87545
}

USA

IAU Colloquium \#151 on Flares and Flashes

Sonneberg, Germany

December 5 - 9, 1994

\begin{abstract}
At Los Alamos National Laboratory we are developing a new imaging sensor which combines high spatial and high temporal resolution over a large area format, while maintaining single-photon counting sensitivity and sustaining a high count rate. The detector is called a microchannel plate with crossed delay line readout, or MCP/CDL. This detector is ideally suited to the observation of weak transient events, such as stellar flares from red dwarf flare stars in our Galaxy. At present we are initiating an experiment with the MCP/CDL detector which will utilize a $30-\mathrm{cm}$ aperture $\mathrm{f} / 7$ telescope to characterize U-band, B-band, and V-band emission from such lowluminosity flare stars, and to search for weak optical transients associated with other astrophysical sources.
\end{abstract}

\section{The MCP/CDL Detector}

Over the past several years the Astrophysics and Radiation Measurements Group at Los Alamos National Laboratory has developed a series of state-of-the-art, singlephoton counting imaging detectors based on microchannel plate (MCP) image intensifiers. The newest version utilizes a crossed delay line for readout of individual photon events, and so it is designated as an MCP/CDL detector. The MCP/CDL utilizes a transmissive S-20 visible photocathode deposited on the inside surface of the vacuum window of the detector. Photoelectrons emitted from this surface are accelerated across a narrow gap to the 40-millimeter active diameter microchannel plates in a z-stack (three plates in series) configuration, providing an electron gain of 107. Each incident photoelectron on the front surface of the stack generates a cloud 
of electrons out the back that passes through a pair of orthogonal helical windings - one wound in the X-direction and the other in the Y-direction. Readout of each photon is accomplished by precisely measuring, at the ends of each delay line, the time difference of arrival of the electron-cloud induced pulses.

The maximum photon counting rate available from the detector is presently one half million per second at $10 \%$ dead time, limited by pileup of events on the 100-ns long delay lines. Within a few months, a new front-end electronics package will boost that rate to ten million photons per second. The demonstrated spatial resolution in our prototype detector is 15 microns, corresponding to a $2000 \times 2000$ pixel circular format. Background count rates from this uncooled detector are approximately 100 counts/s at room temperature, out of the available $10^{6}$ counts/s available overall from the detector.

The output of the detector's electronics is in digital format. Photon events are stored as $X$ and $Y$ positions plus a time tag. This enables us to reconstruct images from photons integrated over long exposures, and to remove any effects of apparent image motion, and to correct for the non-uniformities associated with each detector. It also permits us to easily examine the time history of the photons arriving at any given pixel. Data are stored on multi-gigabyte disk pedestals during observations, and then transferred to mass storage devices for later analysis.

\section{Application to Flare Star Observations}

This detector is well suited for the observation and characterization of magneticallyactive red dwarf flare stars which, in addition to their quiescent output, randomly emit copious quantities of broadband electromagnetic radiation. Observations are typically made in the U-band or B-band to provide the greatest contrast between quiescent and flare output. These flare stars are relatively common in the Galaxy, with an estimated density of around 0.01 per cubic parsec(1) and more recent calculations suggesting that the density is somewhat higher(2). A typical value of U-band energy released in a flare is $10^{32}$ ergs. Previous observations have shown a relationship between the rate of flaring and the energy released in the flare(3). That relationship is

$$
\mathrm{R}\left(>\mathrm{E}_{\mathrm{u} 32}\right)=10^{-2}\left(\mathrm{E}_{\mathrm{u} 32}\right)^{-0.75}
$$

Where $\mathrm{R}$ is the rate $\left(\mathrm{hr}^{-1}\right)$ of flares with energy greater than $\mathrm{E}_{\mathrm{u} 32}$, and the quantity $\mathrm{E}_{\mathrm{u} 32}$ is defined as the U-band energy in units of $10^{32}$ ergs. From this relationship it is evident that the weaker the flare energy one can observe, the higher the rate at which those flares will be observed, and the best statistical results will be obtained from a detector which can image very weak flares from low luminosity flare stars. 
Under moonless and cloudless observing conditions at viewing angles near the zenith, the background photon count rates in the U-band correspond to an average of a few tenths of a count per pixel per second. This is equivalent to continuously monitoring the quiescent U-band output of a 19th magnitude star in each pixel. A flare event which generates photons at a rate that is statistically significant against this background, for example one photon/second/pixel will contribute to our survey.

The minimum detectable energy of a flare is a function of distance; as an example, if the MCP/CDL can detect a flare of $2 \times 10^{30}$ ergs at a distance of 100 parsecs (which corresponds to recording 20 photons at the focal plane of the telescope), then it can detect one of $2 \times 10^{28}$ ergs at 10 parsecs. The expected flare rate seen by our detector is obtained by integrating out along the 1.1 degree cone of the field of view of the telescope. B-band calculations done for our new 30-cm aperture telescope, assuming a 20-photon observational threshold and event scaling according to equation (1), show that flares should be detected at a rate of several per hour, when viewing a general galactic field.

\section{Current Status of the Experiment}

At the present time we have one working version of the MCP/CDL detector. As result of a degraded photocathode response due to some suspected high-voltage breakdowns over time, this unit is sufficiently insensitive that it cannot be used for routine flare star survey sessions. A few images, however, were obtained with this unit in the summer of 1994 using a 15-cm aperture refractor telescope and a 7-inch aperture Questar telescope. These images have provided verification of background photon counting rates, as well as a data base with which develop the algorithms necessary for image reconstruction. One example of a V-band image obtained in the July observation is shown in Figure 1. The exposure was integrated over approximately four minutes from the fixed refractor telescope. The resulting raw image shows streaks corresponding to the apparent motion of the stars. This motion has been removed in software, and the integrated (point) stellar images are as shown in Figure 1. The two remaining streaks in this image were generated from fixed hot spots in the detector that were spread out during image reconstruction.

At the present time we also have a small amount of data obtained in the U-band, taken with the 7-inch aperture Questar telescope. It is sufficient to verify background photon counting rates for comparison to theoretical values. We do not yet have sufficient data to begin any search for flare events. That search will begin with our acquisition of a new detector and telescope in early 1995. 


\section{Observation Schedule}

We are presently awaiting delivery of a new telescope that has been designed specifically for the flare star project with this MCP/CDL detector. The modified Ritchey-Chretien optical configuration has been designed to provide a flat-field image over the 40-millimeter diameter of the MCP/CDL detector for the $\mathrm{U}, \mathrm{B}$, and $\mathrm{V}$ bands. Its aperture is $30 \mathrm{~cm}$ and the focal ratio is $\mathrm{f} / 7$. These values have been selected in order to match the maximum photon counting rate capability of the MCP/CDL electronics, where it operates at highest efficiency. This telescope is under construction, and due for delivery in January, 1995. We also expect delivery of a new MCP/CDL detector from our subcontractor who does the microchannel plate preparation, photocathode deposition, and vacuum sealing of the units. The anticipated delivery of this new unit is in March, 1995. Observations will begin soon thereafter, at a remote site in the Jemez mountains located thirty five miles from Los Alamos National Laboratory.

\section{References}

(1) Mirzoyan, L.V., (1990) IAU Symposium \#137 on Flare Stars in Star Clusters, Associations, and the Solar Vicinity, 5.

(2) This Conference; Poster Paper \#14, L.V. Mirzoyan, and Poster Paper \#25, N.I. Shakovskaya.

(3) Gershberg, R.E., and Shakovskaya, N.I., (1983) Astrophysics and Space Science, 95235. 


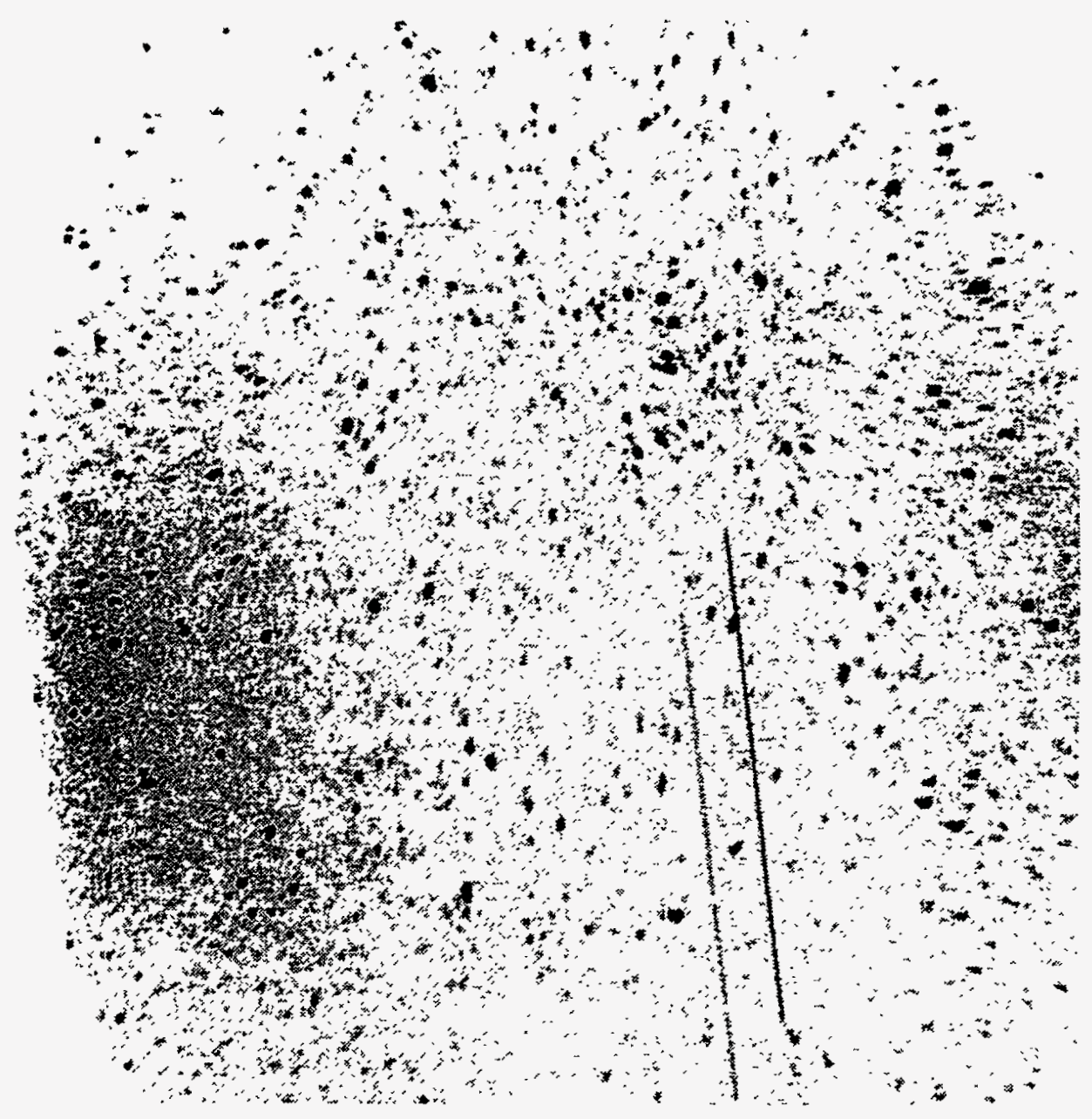

Figure 1. Stellar image from the vicinity of the constellation Aquila obtained with the MCP/CDL detector. The exposure time was four minutes with a fixed $15-\mathrm{cm}$ aperture refractor telescope. Stellar motion has been removed in software leaving two detector hot-spot streaks (see text). 